\title{
Unveiling the Molecular Structure of Pulmonary Surfactant Corona on Nanoparticles
}

Qinglin $\mathrm{Hu}^{\dagger,+}$ Xuan Bai, ${ }^{\dagger, \ddagger}$ Guoqing $\mathrm{Hu}^{,},{ }^{\dagger,+}$ and Yi Y. Zuo ${ }^{*, \S, \| \odot ~}$

${ }^{\dagger}$ The State Key Laboratory of Nonlinear Mechanics (LNM), Institute of Mechanics, Chinese Academy of Sciences, Beijing 100190, China

${ }^{\ddagger}$ School of Engineering Science, University of Chinese Academy of Sciences, Beijing 100049, China

${ }^{\S}$ Department of Mechanical Engineering, University of Hawaii at Manoa, Honolulu, Hawaii 96822, United States

"Department of Pediatrics, John A. Burns School of Medicine, University of Hawaii, Honolulu, Hawaii 96826, United States

\author{
Supporting Information
}

ABSTRACT: The growing risk of human exposure to airborne nanoparticles (NPs) causes a general concern on the biosafety of nanotechnology. Inhaled NPs can deposit in the deep lung at which they interact with the pulmonary surfactant (PS). Despite the increasing study of nano-bio interactions, detailed molecular mechanisms by which inhaled NPs interact with the natural PS system remain unclear. Using coarse-grained molecular dynamics simulation, we studied the interaction between NPs and the PS system in the alveolar fluid. It was found that regardless of different physicochemical properties, upon contacting the

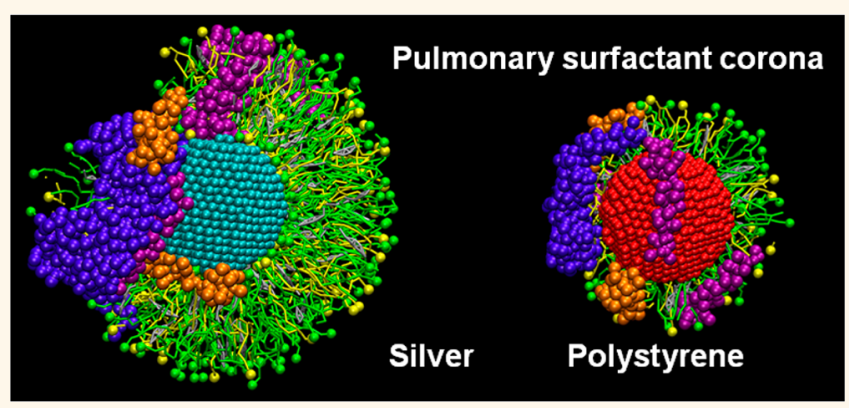
PS, both silver and polystyrene NPs are immediately coated with a biomolecular corona that consists of both lipids and proteins. Structure and molecular conformation of the PS corona depend on the hydrophobicity of the pristine NPs. Quantitative analysis revealed that lipid composition of the corona formed on different NPs is relatively conserved and is similar to that of the bulk phase PS. However, relative abundance of the surfactant-associated proteins, SP-A, SP-B, and SP$\mathrm{C}$, is notably affected by the hydrophobicity of the NP. The PS corona provides the NPs with a physicochemical barrier against the environment, equalizes the hydrophobicity of the pristine NPs, and may enhance biorecognition of the NPs. These modifications in physicochemical properties may play a crucial role in affecting the biological identity of the NPs and hence alter their subsequent interactions with cells and other biological entities. Our results suggest that all studies of inhalation nanotoxicology or NP-based pulmonary drug delivery should consider the influence of the PS corona.

KEYWORDS: pulmonary surfactant, nanoparticle, biomolecular corona, molecular dynamics, nanotoxicology

U nderstanding interactions between nanoparticles (NPs) and living organisms is of crucial importance for advancing biomedical applications such as NPbased drug delivery and for regulating the environmental, health, and safety (EHS) issues of nanotechnology. ${ }^{1}$ With a larger surface free energy than the bulk material, NPs immediately adsorb biomolecules upon contacting biological specimens to form the so-called biomolecular corona. ${ }^{2-4}$ Biophysicochemical properties of this corona, instead of the pristine NPs, determines the biological identity of the NPs when further interacting with the biological system. ${ }^{4}$ Numerous studies have shown that the biomolecular corona alters the biodistribution, bioreactivity, and biopersistence of NPs, ${ }^{1,5}$ thus resulting in differential cellular uptake and cytotoxicity of the NPs. $^{6-9}$
To date, a majority of studies in the biomolecular corona focuses on the protein corona. Upon intravascular administration of NPs, thousands of plasma proteins including the serum albumin are found to adsorb onto the NP surface to form the protein corona. ${ }^{3}$ Available experimental data reveal that the protein corona is composed of an inner layer of tightly bound molecules called the "hard corona" and an outer layer of "soft corona" consisting of molecules only loosely attached to the NP surface. ${ }^{10,11}$ Whereas molecules in the soft corona undergo dynamic adsorption-desorption cycles and thus only have a short lifetime of seconds or less, the hard corona is

Received: March 17, 2017

Accepted: May 25, 2017

Published: May 25, 2017 

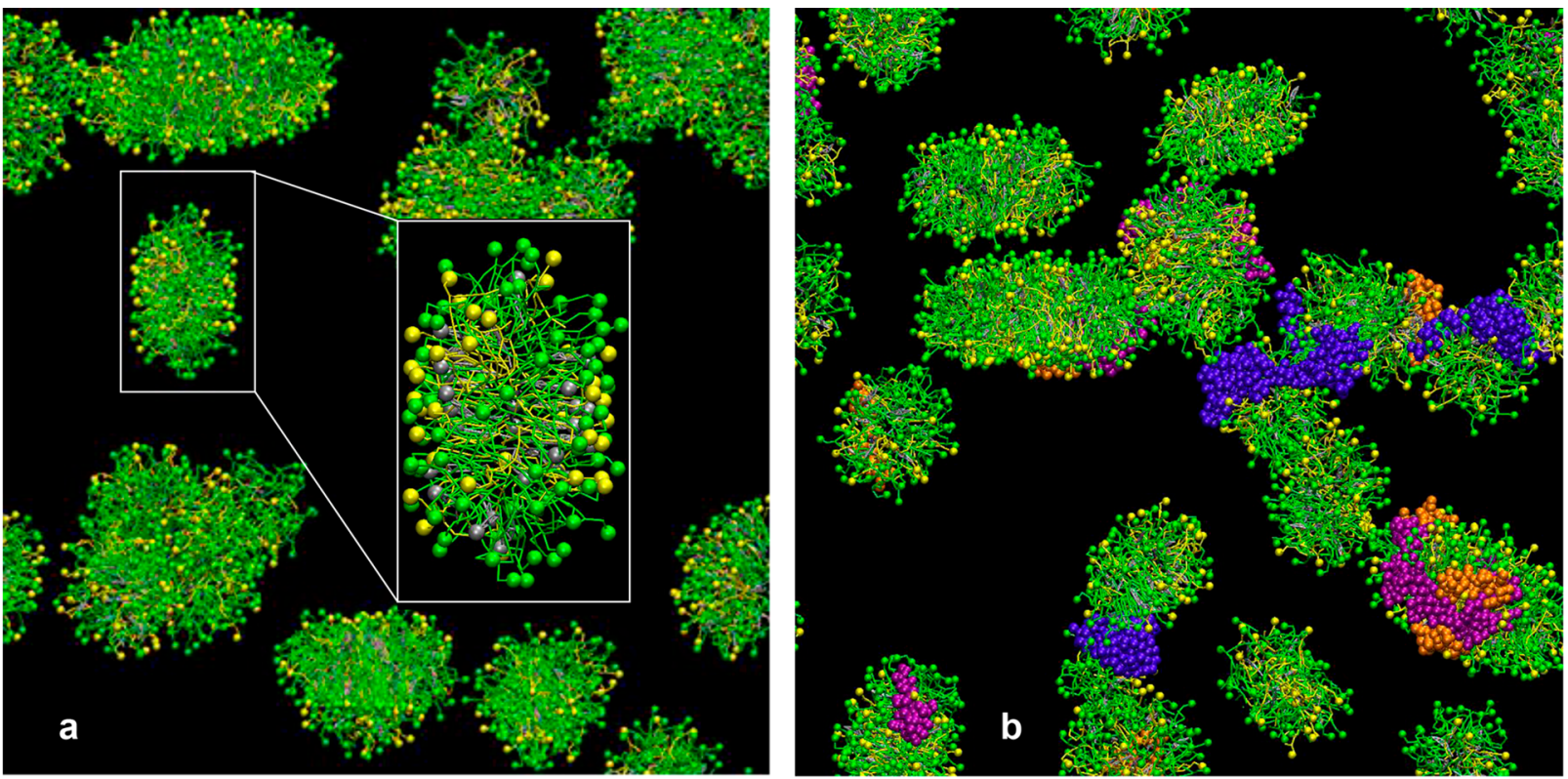

Figure 1. CGMD simulations of pulmonary surfactant (PS) lipids without (a) and with (b) surfactant-associated proteins self-assembled in the bulk aqueous phase. The PS system contains 1400 DPPC (in green), 600 POPG (in yellow), 500 cholesterol (in silver), 4 SP-A fragments (in violet), 10 mini-B (in orange), and $10 \mathrm{SP}-\mathrm{C}$ peptides (in purple) in a cubic space of $40 \times 40 \times 40 \mathrm{~nm}^{3}$. For clarity, water and ions are not shown. The lipid contents correspond to a weight concentration of $0.044 \mathrm{mg} / \mathrm{mL}$. Inset in (a) shows the detailed structure of a micelle.

relatively stable and has a much longer lifetime up to hours. ${ }^{11}$ Proteomic analysis with mass spectrometry has further identified that compositions of the protein corona depend on physicochemical properties of the NPs such as their size, charge, and hydrophobicity. ${ }^{12,13}$

Very recently, the study of protein corona has been generalized by considering portals of particle entry other than intravascular injection. Indeed among all potential portals of entering the human body, the respiratory system is the most susceptible to particle invasion due to its large surface area in direct contact with the environment. ${ }^{14}$ With their small size, a large portion of inhaled NPs can penetrate the respiratory tracts and deposit in the deep lung, where the NPs first interact with the pulmonary surfactant (PS) lining layer of alveoli and develop the so-called PS biomolecular corona. ${ }^{15-17}$

The PS is composed of approximately $90 \%$ lipids, including various phospholipids and cholesterol, and $10 \%$ proteins (named SP-A, SP-B, SP-C, and SP-D) by weight. ${ }^{18}$ Among these four surfactant-associated proteins, SP-B and SP-C are small hydrophobic membrane proteins that are essentially important for reducing alveolar surface tensions and thus indispensable for sustaining normal respiratory physiology. ${ }^{19}$ SP-A and SP-D are large hydrophilic proteins and members of the collectin family, which play an important role in innate host defense by binding inhaled particles and pathogens. ${ }^{20,21}$

The importance of understanding the PS corona is at least two-fold. First, the process of forming the biomolecular corona on inhaled NPs upon contacting the PS system represents the initial nano-bio interactions in the lungs. Hence, formation of the PS corona may interfere with the physiological function of the endogenous PS, thus posting a significant nanotoxicological impact on the respiratory system. For example, recent experimental evidence has convincingly shown that interactions with NPs can adversely affect the biophysical function of natural PS in reducing the surface tension. ${ }^{15,16,22-26}$ Second, because the PS corona is formed at the initial biological barrier in the lungs, its biophysicochemical properties, rather than those of the pristine NPs, determine the subsequent biological identity and fate of the inhaled NPs, ${ }^{4}$ such as their retention at the alveolar space, interaction with epithelial cells, clearance by macrophages, and translocation to the systemic circulation and other organs. Along this line, plenty of experimental data have demonstrated that preincubating various NPs (e.g., singlewalled carbon nanotubes, ${ }^{27}$ polystyrene, ${ }^{28}$ and silica NPs $)^{29}$ in the PS-containing bronchoalveolar fluid significantly alters the NP uptake by alveolar epithelial cells and macrophages.

In spite of the importance of the PS biomolecular corona, relevant studies are still scarce. Available experimental evidence of the PS corona mainly relies on mass spectrometry, ${ }^{17,27,30,31}$ which can only provide knowledge of chemical composition of the corona rather than its molecular conformation. Consequently, the general mechanisms by which the PS corona is formed remain unclear. Here, we use coarse-grained molecular dynamics (CGMD) simulations to study the structure and molecular conformation of the PS corona formed on NPs of representative surface properties. We first established the detailed CGMD model of the natural PS assembly system in the alveolar fluid. With this model system, we conducted the molecular scale simulation of the PS corona formed on two commonly studied NPs, that is, the hydrophilic silver NP (AgNP) and the hydrophobic polystyrene NP (PST-NP). Our simulations suggest that regardless of different surface properties of the NPs, upon contact with the bulk phase PS in the alveolar fluid, the NPs are instantly wrapped with a biomolecular corona that consists of both lipids and proteins. The composition, structure, and conformation of biomolecules in the PS corona are regulated by surface properties of the NP. Our simulation results shed light on understanding the molecular mechanism of nano-bio interactions in the respiratory system. 


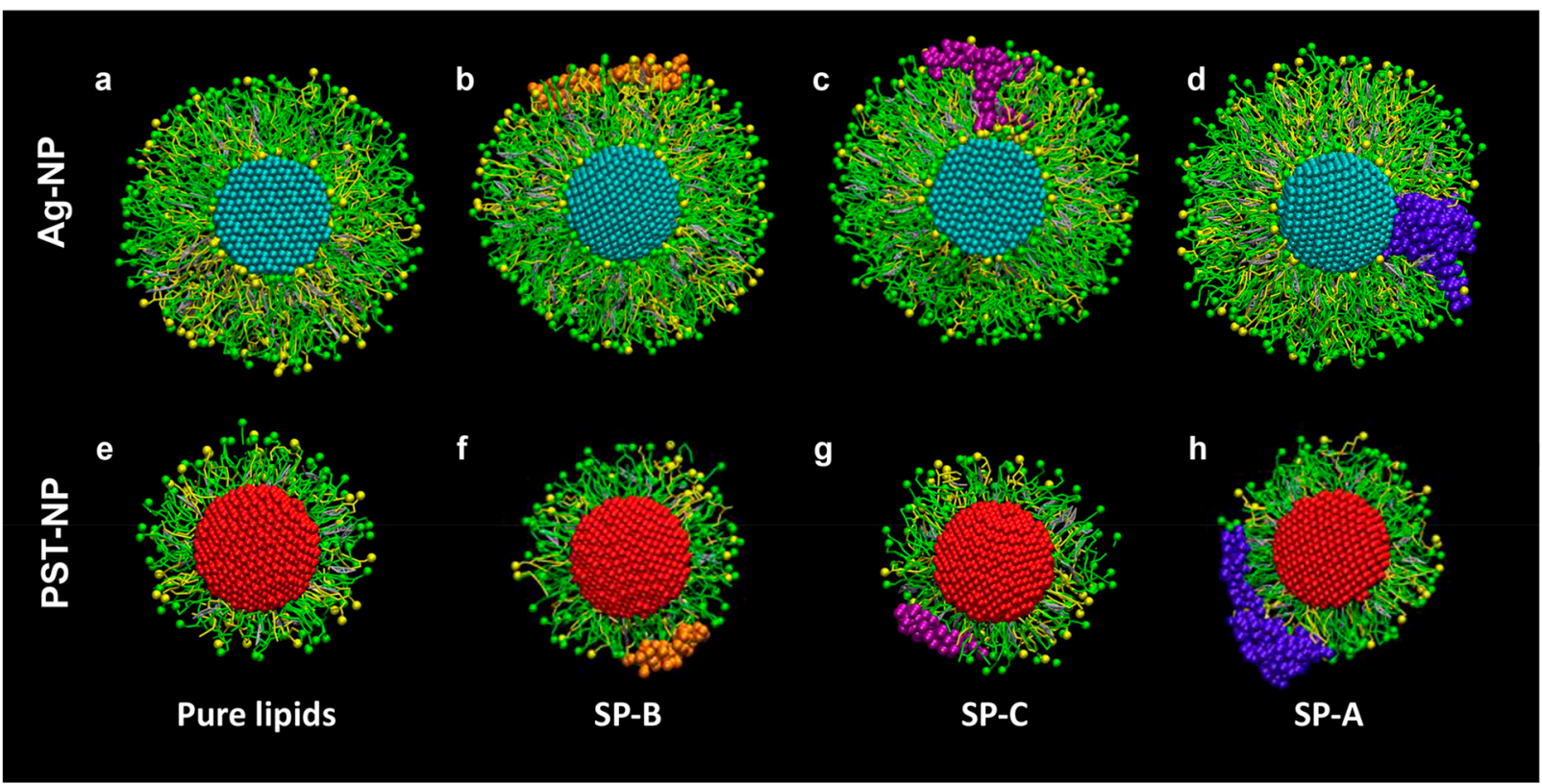

Figure 2. Chemical compositions and molecular structures of the PS corona on two representative NPs: (a-d) silver (Ag-NP) and (e-h) polystyrene (PST-NP). The Ag-NP and PST-NP are shown in cyan and red, respectively. DPPC molecules are shown in green, POPG in yellow, and cholesterol in silver. The three surfactant-associated proteins: SP-B is shown in orange, SP-C in purple, and SP-A in violet.

\section{RESULTS AND DISCUSSION}

Self-Assembly of PS Molecules in the Aqueous Phase. Before introducing NPs, we first simulate the micellar ultrastructure of natural PS self-assembled in the aqueous phase of the alveolar fluid. Figure 1a shows the self-assembled structure with pure lipid components without surfactantassociated proteins (the dynamic process of lipid self-assembly can be found in Video S1 of the Supporting Information). Lipids in our model system include dipalmitoylphosphatidylcholine (DPPC), palmitoyloleoylphosphatidylglycerol (POPG), and cholesterol at a molecular ratio of 14:6:5. Although simpler than the natural PS, lipids in this model system represent the major lipid components of PS, retaining the diversity of zwitterionic (PC), anionic (PG), and neutral (cholesterol) lipids, with both disaturated (dipalmitoyl) and unsaturated (palmitoyloleoyl) acyl chains. ${ }^{18,32}$ Our previous MD simulations of PS monolayers have proven the accuracy of this model system in comparison with topographic structures of natural PS monolayers obtained with atomic force microscopy. ${ }^{16}$

As shown in Figure 1a, without surfactant proteins, molecules of DPPC (in green) and POPG (in yellow) are self-assembled into micelles with the hydrophobic tails of the phospholipids shielded inside a shell made of hydrophilic head groups. This structure minimizes the system energy by preventing water molecules from contacting the hydrophobic moieties of the amphiphilic phospholipids. As detailed in the inset, cholesterol molecules (in silver) are found to be embedded within the micellar structure, with its hydroxy groups in contact with the phospholipid head groups and its alkyl groups in contact with the phospholipid tails. This finding of MD simulations is consistent with experimental prediction that cholesterol selectively interacts with hydrocarbon chains of phospholipids. ${ }^{18,33}$ Consequently, a physiological level of cholesterol helps solidify the PS membranes, but a supraphysiological level of cholesterol deteriorates the biophysical function of PS by fluidizing the surfactant vesicles and films. ${ }^{18,33}$
Figure $1 \mathrm{~b}$ shows the self-assembled PS ultrastructure with the addition of three membrane-binding surfactant proteins, such as SP-B, SP-C, and SP-A (the dynamic process of PS selfassembly can be found in Video S2 of the Supporting Information). It is found that all three proteins promote fusion of the unilamellar micelles into larger multilamellar micelles, in good agreement with experimental observations of the ultrastructure of protein-containing natural PS. ${ }^{15,34}$ Specifically, it is found that the hydrophobic SP-B (in orange) and SP-C (in purple) mainly assume transmembrane positions, whereas the hydrophilic SP-A (in violet) locates primarily at the intermembrane space to bridge individual micelles. In general, results of our MD simulations are in good agreement with the current molecular understanding and predication of lipidprotein interactions in natural PS vesicles and films. ${ }^{18,32}$

Structure and Conformation of Individual Biomolecular Components in the PS Corona. Once establishing the model system of bulk phase PS in the alveolar fluid, we simulated the formation of the PS corona on two commonly studied NPs with representative characteristics, that is, the hydrophilic silver NP (Ag-NP) and the hydrophobic polystyrene NP (PST-NP). Ag-NPs are widely used in many industrial applications such as electronics, biosensing, food industry, cosmetics, and medical devices. ${ }^{35}$ PST-NPs are commonly used for biomedical applications and as a model particle for studying nanotoxicology. ${ }^{8,11,12,22}$ Both the Ag-NP and PST-NP were simulated as $5 \mathrm{~nm}$ spheres with a negative surface charge density set as $0.06 \mathrm{e} / \mathrm{nm}^{2}$, based on the potentiometric and conductometric titrations by Stone-Masui and Watillon. ${ }^{36}$ The effect of particle size on the formation of the PS corona was also studied. These results are summarized in Figure S1 of the Supporting Information (SI). It can be seen that the structure of the lipid corona formed on NPs of two sizes (i.e., 5 and $15 \mathrm{~nm}$ ) does not differ significantly, except that the process of corona formation on the smaller NP is quicker than that on the larger NP. Therefore, in the rest of the study, we only focused on NPs of $5 \mathrm{~nm}$ in diameter. 


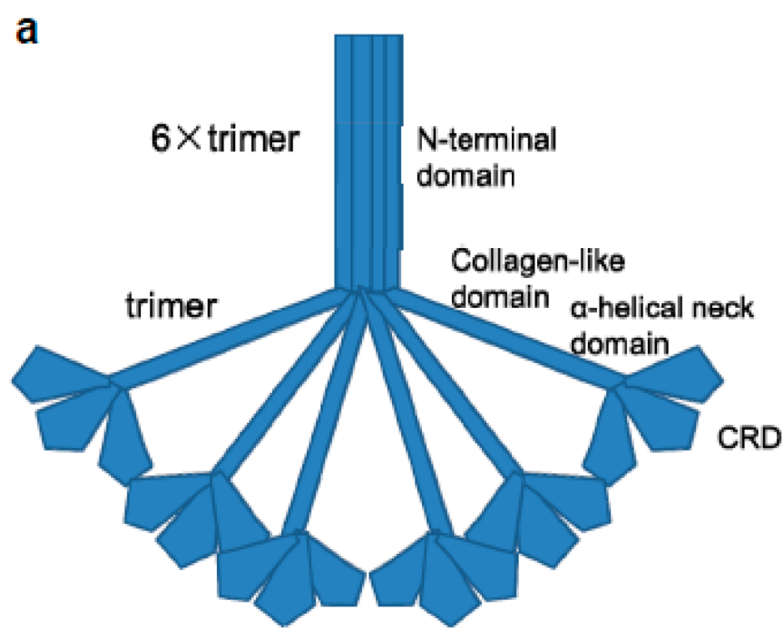

SP-A structure

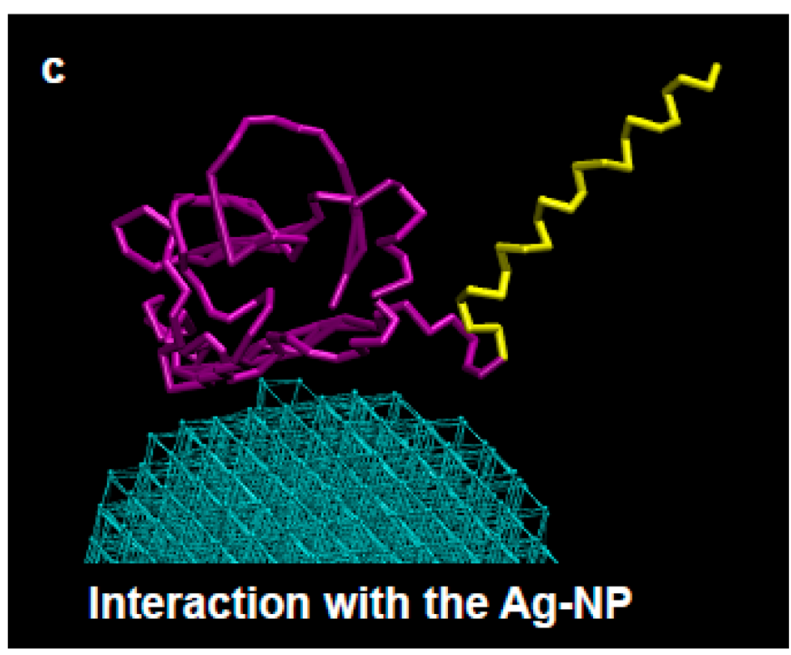

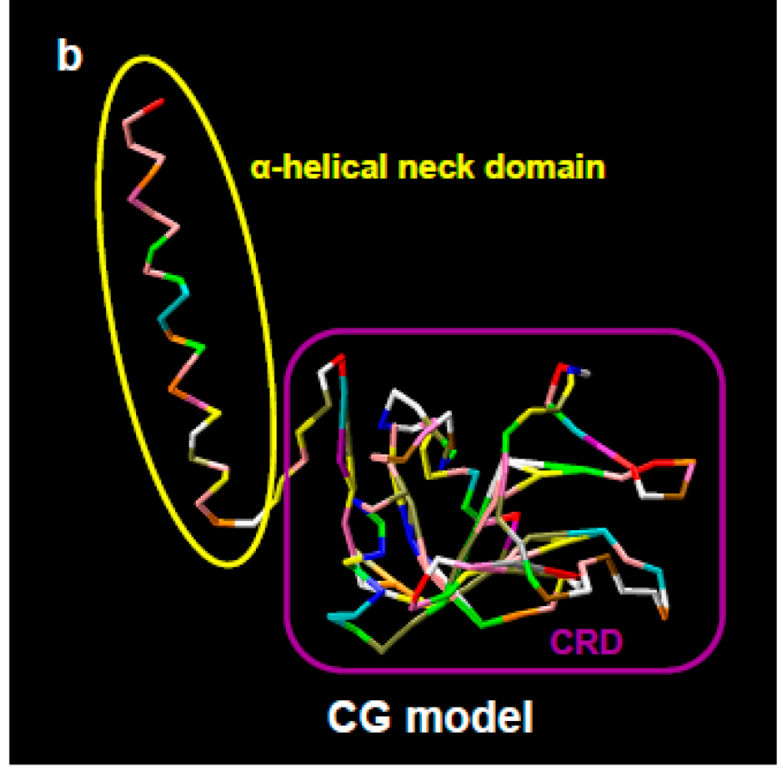

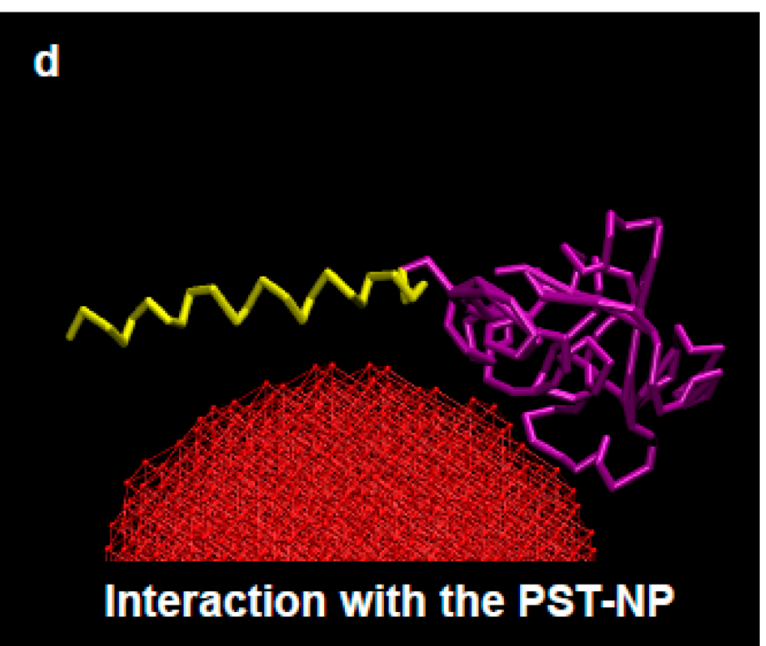

Figure 3. SP-A interaction with NPs of different surface properties. (a) Schematic of the SP-A molecule, which is a flower bouquet-like octadecamer assembled from six trimers, with each monomer consisting of a carbohydrate recognition domain (CRD), an $\alpha$-helical neck domain, a collagen-like domain, and an N-terminal cysteine-rich domain. (b) Licorice representation of the CGMD model of the SP-A fragment simulated in this study. The SP-A fragment contains the CRD and the $\alpha$-helical neck domain. (c,d) Molecular conformation of the SP-A fragment bound to the Ag-NP and PST-NP, respectively.

Figure 2 shows the simulated structures of the PS corona on the Ag-NP and the PST-NP. For clarity, structure of the lipid corona, and the molecular conformation of three surfactantassociated proteins, the hydrophobic SP-B (in orange) and SP$\mathrm{C}$ (in purple) and the hydrophilic SP-A (in violet), are individually studied and demonstrated in the cross-sectional view of the corona.

First, it is clear that the PS corona formed on both the AgNP and PST-NP is a lipoprotein complex that consists of both lipids and proteins. The specific structure of the corona and its molecular conformation are affected by the hydrophobicity of the NP. In general, lipid molecules in the corona assume a bilayer conformation on the hydrophilic Ag-NP (Figure 2a) but assume a monolayer conformation on the hydrophobic PSTNP (Figure 2e). The existence of surfactant proteins does not significantly vary the basic conformation of lipid molecules in the corona.

Second, the two hydrophobic surfactant proteins, SP-B and SP-C, appear to mediate the formation of PS corona by directly interacting with the lipids in corona. In the corona formed on the Ag-NP, SP-B interacts peripherally with the lipid corona and orients in parallel to the lipid bilayer (Figure $2 \mathrm{~b}$ ), whereas the helical fragment of SP-C adopts a transmembrane orientation (Figure 2c), similar to the generally accepted molecular orientations of these two hydrophobic proteins in PS membranes. ${ }^{32}$ In the corona formed on the PST-NP, due to the monolayer conformation of the corona, both SP-B (Figure 2f) and SP-C (Figure 2g) assume an orientation largely in parallel with the lipid monolayer.

Both SP-B and SP-C are hydrophobic, positively charged ( $\mathrm{pI}$ $=8-9)$, and tightly associated with the surfactant membrane. ${ }^{19,32}$ However, SP-C is significantly more hydrophobic than SP-B. As shown in Figure S2 of the SI, SP-C is enriched in valine, leucine, and isoleucine, which makes SP-C one of the most hydrophobic proteins in the proteome. The 13th to 28th amino acid residues of SP-C all consist of these three hydrophobic amino acids. They form an $\alpha$-helix structure, with its length equivalent to the thickness of a phospholipid 

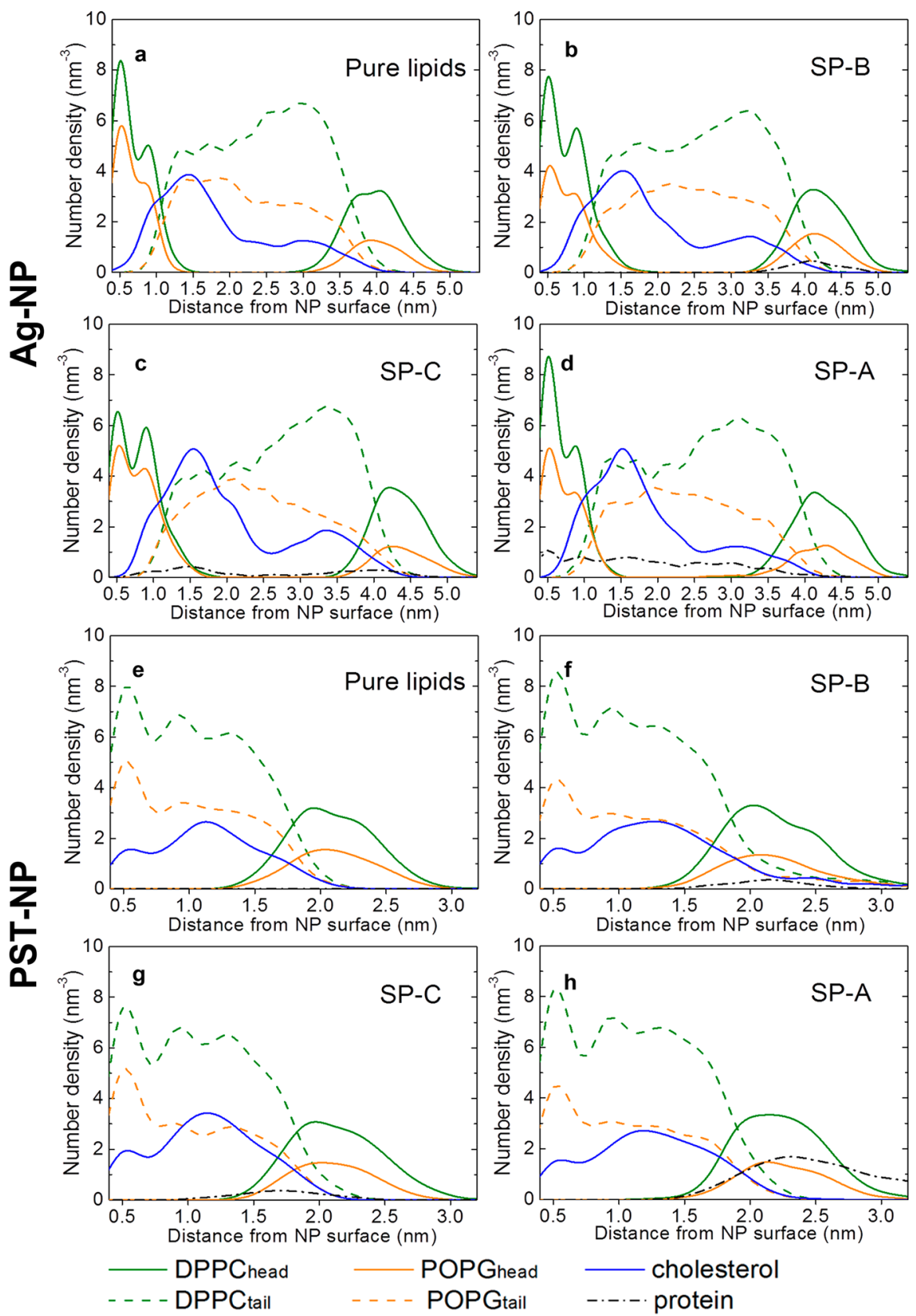

Figure 4. Number density of different PS components as a function of their distances from the surface of two representative NPs: (a-d) silver (Ag-NP) and (e-h) polystyrene (PST-NP). (a-h) Analyses of the individual simulation cases shown in Figure 2, i.e., (a,e) analyze the pure lipids; (b,f) analyze the lipids with SP-B; (c,g) analyze the lipids with SP-C; (d,h) analyze the lipids with SP-A.

bilayer. Therefore, SP-C tends to form lipid-spanning structures. SP-B, on the other hand, is less hydrophobic than SP-C and does not form transmembrane structures as SP-C does. It has been estimated that in the membrane and film of natural PS, SP-B likely interacts with the phospholipid molecules through the main axis of its amphipathic helical segments orientated in parallel to the plane of the lipid bilayers; ${ }^{37}$ however, SP-C, being an even more hydrophobic protein, most likely assumes a transmembrane orientation. Our MD simulations suggest that each of these proteins in the PS corona may assume a molecular orientation similar to that in the PS membranes. Consequently, both proteins behave as a mediator to regulate and promote the formation of the PS corona on the NP.
Third, the hydrophilic surfactant protein, SP-A, appears to be also associated with the PS corona, albeit to a lesser extent than the hydrophobic proteins. Our simulations show that the SP-A molecule partially contacts the Ag-NP (Figure 2d), while it orients nearly in parallel with the lipid monolayer in the corona formed on the PST-NP (Figure 2h). To better understand the molecular orientation of SP-A in the corona and its functional moiety interacting with the NP, we simulated the detailed interaction between SP-A and NPs. As shown in the schematic in Figure 3a, the SP-A molecule is a flower bouquet-like octadecamer assembled from six trimers, with each monomer consisting of a carbohydrate recognition domain (CRD), an $\alpha$ helical neck domain, a collagen-like domain, and an N-terminal cysteine-rich domain. ${ }^{21,38}$ This molecular structure is too complicated to be fully simulated with the current $\mathrm{MD}$ 

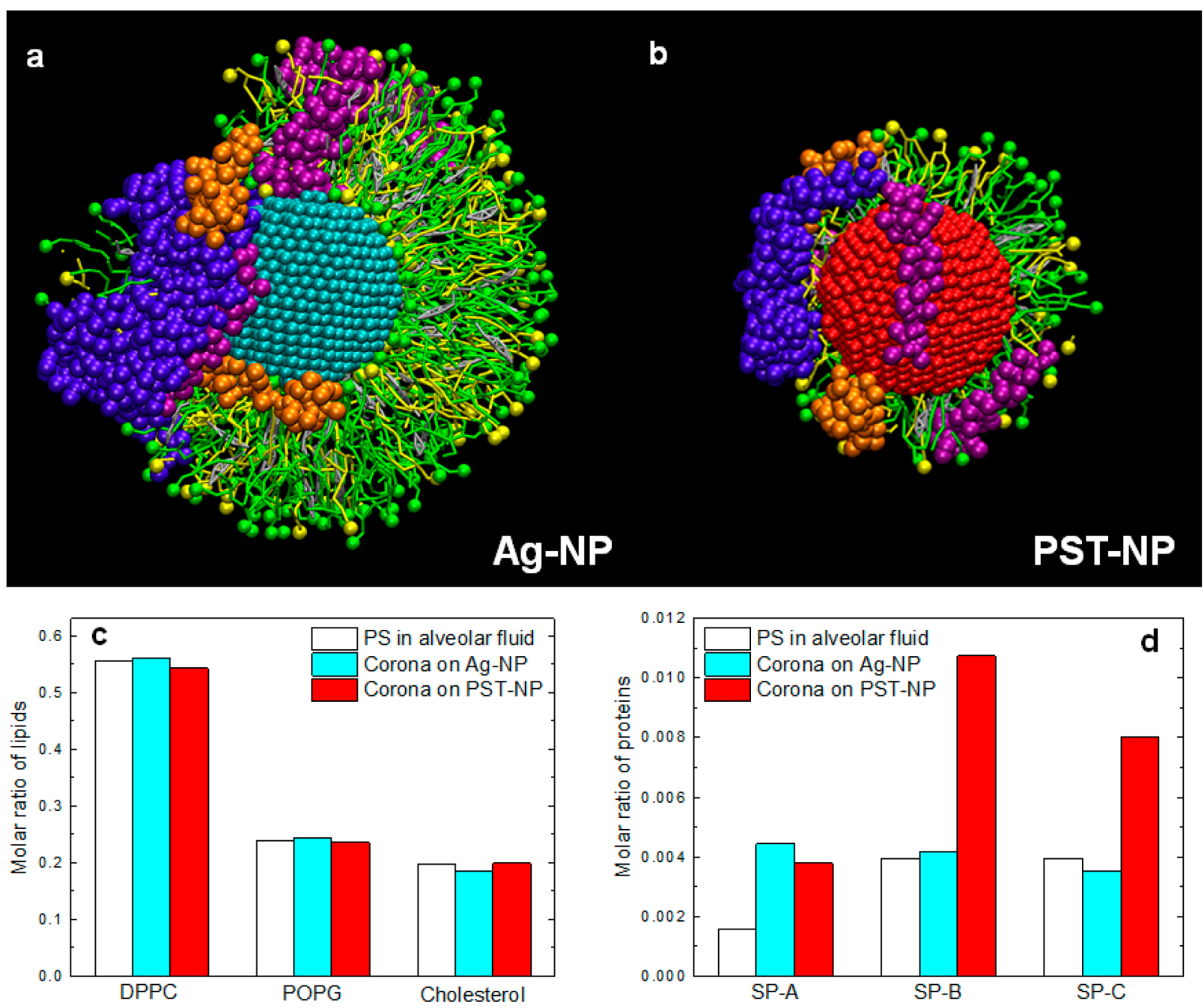

Figure 5. Chemical composition of the PS corona formed on two representative NPs: (a) silver (Ag-NP, shown in cyan) and (b) polystyrene (PST-NP, shown in red). (c,d) Quantitative comparison of molar ratios of lipids and proteins in the PS corona with those in the bulk phase PS.

simulation techniques. Hence, we simulated a SP-A fragment that contains the CRD and the $\alpha$-helical neck domain because it is widely accepted that the CRD is responsible for SP-A's affinity to multiple ligands and thus facilitates its host defense function. $^{21,38}$ Figure $3 \mathrm{~b}$ demonstrates the CG model of the SPA fragment used in our simulations. It consists of a more hydrophilic CRD (containing 87 hydrophilic beads) and a less hydrophilic $\alpha$-helical neck domain (containing 36 hydrophilic beads). Figure $3 c, d$ shows the detailed molecular orientation of the SP-A fragment bound to the Ag-NP and PST-NP, respectively. It can be seen that regardless of the physicochemical properties of the NP, the SP-A fragment binds exclusively to the NP surface via the CRD. The different molecular orientation of the SP-A molecule in the corona is caused by different tilting angles of the $\alpha$-helical neck domain on the AgNP and PST-NP. The more hydrophilic CRD tends to adsorb onto the hydrophilic surface of the Ag-NP in competition with the hydrophilic heads of the phospholipids, whereas the less hydrophilic neck domain adopts a transmembrane orientation. This finding reinforces the current understanding of molecular mechanism by which SP-A recognizes inhaled NPs, thus stimulating phagocytosis and macrophage clearance of these particles. ${ }^{39-41}$
To quantitatively describe the molecular structure of the PS corona shown in Figure 2, Figure 4 shows the distribution density of different PS components as a function of their distances from the NP surface, corresponding to the individual cases $(a-h)$ shown in Figure 2. For the Ag-NP, the distribution of phospholipid head groups shows two major peaks around 0.5 and $4.1 \mathrm{~nm}$, with all phospholipid tail groups and cholesterol distributed between these two peaks (Figure 4a). This clearly indicates a bilayered molecular conformation. In contrast, the distribution of phospholipid head groups for the PST-NP shows only one peak around $2.1 \mathrm{~nm}$ away from the NP surface (Figure 4e), thus indicating a monolayer conformation. All three surfactant-associated proteins (i.e., SP-B, SP-C, and SP-A) are found to be associated with the corona but do not significantly disturb the lipid conformation of the corona.

PS Corona Formed on Representative NPs: Silver and Polystyrene. After investigating the detailed structure and conformation of individual biomolecular components in the corona (Figures 2-4), here we simulated the complete PS corona formed on the two representative NPs, that is, the AgNP and PST-NP. Due to complexity of the system, we further increased the simulation time from 0.75 to $2 \mu$ s to ensure the reach of equilibrium. Figure 5a,b shows the simulation results of 
Table 1. Physicochemical Properties of the Silver Nanoparticle (Ag-NP) and the Polystyrene Nanoparticle (PST-NP) Modified by the Coated Pulmonary Surfactant (PS) Corona

\begin{tabular}{|c|c|c|c|}
\hline & diameter $(\mathrm{nm})$ & hydrophobicity $(0-1)^{a}$ & surface density of the phosphate group $\left(\mathrm{nm}^{-2}\right)^{b}$ \\
\hline \multirow[t]{2}{*}{ Ag-NP/with PS corona } & 5.0 & 0.00 & 0.00 \\
\hline & 14.8 & 0.37 & 0.45 \\
\hline \multirow[t]{2}{*}{ PST-NP/with PS corona } & 5.0 & 1.00 & 0.00 \\
\hline & 9.8 & 0.41 & 0.39 \\
\hline
\end{tabular}

${ }^{a}$ The relative hydrophobicity of the NP (with/without the PS corona) was characterized by the proportion of hydrophobic beads on the NP surface, with zero representing fully hydrophilic and unity representing fully hydrophobic. ${ }^{b}$ The surface density of the phosphate group on the NP was evaluated by $N /\left(\pi D^{2}\right)$, where $N$ is the number of PO4-named beads on the outer surface of the corona, and $\pi D^{2}$ is the surface area of the corona.

the PS corona formed on the Ag-NP and PST-NP, respectively. Figure $5 \mathrm{c}, \mathrm{d}$ shows the quantitative analysis of molar ratios of lipids and proteins in the corona in comparison with those of the bulk phase PS in the alveolar fluid. The molar ratio is defined as the ratio of the number of molecules of each lipid and protein components in the corona to the total number of molecules in the corona. Quantitative analysis of the PS corona in terms of the number density of different PS components is shown in Figure S3 in the SI. The dynamic process of forming the PS corona on the Ag-NP and the PST-NP can be found in the Video S3 and Video S4 in the SI. We have also studied the dependence of our results on the simulation conditions, such as temperature and lipid and protein concentrations. These results can be found in Figures S4-S6 in the SI.

It is found that regardless of different surface properties of the NPs, the major lipid components (i.e., DPPC, POPG, and cholesterol) in the corona are relatively conserved and do not differ significantly from the lipid composition in the bulk phase PS (Figure 5c). However, it appears that the composition of surfactant-associated proteins in the corona is significantly different from that in the bulk phase PS and is apparently mediated by the surface properties of the NP. As shown in Figure 5d, the molecular ratio of SP-A in both coronas nearly doubles that in the bulk phase PS. In contrary to SP-A, which has a similar molar ratio in the coronas for both the Ag-NP and PST-NP, contents of SP-B and SP-C are clearly regulated by the surface properties of the NP. Whereas the molar ratios of SP-B and SP-C in the corona of the Ag-NP are similar to those in the bulk phase PS, these two hydrophobic proteins are found to be significantly enriched in the corona of the PST-NP, indicating a strong tendency of selective protein adsorption mediated by hydrophobic interactions.

Our simulation results are in good agreement with recent lipidomic and proteomic characterization of the PS corona using high-resolution mass spectrometry. ${ }^{17,27,30}$ Raesch et al. found that the lipid composition of the biomolecular corona formed on NPs incubated with the porcine bronchoalveolar lavage fluid (BALF) was relatively conserved, without significant difference from the BALF in the relative abundance of lipid species. ${ }^{17}$ Similar experimental results were reported by Kapralov et al. $^{27}$ After recovering single-walled carbon nanotubes (SWCNTs) from the mouse BALF with the pharyngeal aspiration model, these researchers found that the biomolecular corona formed on the SWCNTs contains major PC and PG components, similar to the lipid profile of the mouse BALF. Our simulations clearly demonstrate that SP-A selectively adsorbs to the NP regardless of its surface properties (Figure $5 d)$. Such results are not unexpected as increasing experimental evidence suggests direct binding between SP-A and NPs. ${ }^{17,42,43}$ Our finding of increasing relative abundance of hydrophobic proteins (SP-B and SP-C) in the corona is also consistent with experimental results by Sund et al. ${ }^{30}$ They found that the SP-B was among the major proteins attached to the surface of NPs incubated with Curosurf, a clinically used porcine surfactant preparation.

Importance of the PS Corona and Its Implications to Nanotoxicology. Numerous studies have shown that it is the biophysicochemical properties of the biomolecular corona, instead of properties of the pristine NPs, that determine the biological identity of the NPs in interacting with the biological system. ${ }^{4}$ As a counterpart of the protein corona formed on NPs entering the blood vessels, the PS corona is acquired immediately and inevitably after NPs enter the respiratory system. Once the PS corona is formed on the NP surface, the biophysicochemical properties of the corona determine the further nano-bio interactions of the NPs, such as their bioavailability, biodistribution, retention, translocation, and clearance.

Table 1 summarizes the major change of biophysicochemical properties of the Ag-NP and PST-NP after acquiring the PS corona. First, the PS corona coated on the pristine NP increases the effective particle size. Such an effect is obviously more significant for small NPs, which appear to be the most proinflammatory particles. ${ }^{44}$ It is found that with the PS corona, diameter of the Ag-NP increases from 5 to $14.8 \mathrm{~nm}$ (Table 1). The lungs are the major organ targeted by prolonged exposure to Ag-NPs. ${ }^{35,45-47}$ Release of free silver ions after Ag-NP dissolution in the biological environment is found to be the primary mechanism of nanosilver toxicity..$^{35,45-47}$ Experimental studies demonstrated that incubating Ag-NPs with DPPC (i.e., the major lipid component of natural PS) attenuates the dissolution of Ag-NPs, inhibits the release of silver ions, ${ }^{48}$ and hence reduces the proinflammatory potential of Ag-NPs in the lungs. ${ }^{44}$ More importantly, such a beneficial effect of the PS corona is most likely nonspecific to Ag-NPs as it was found that incubation of zinc oxide nanowires with Curosurf also reduces particle dissolution in the biological environment. ${ }^{49}$ Hence, the PS corona, in general, may reduce the proinflammatory potential of NPs by forming a physicochemical barrier against the biological environment.

Second, the PS corona coated on the pristine NP appears to equalize its hydrophobicity, that is, making the intrinsically hydrophilic NP more hydrophobic while making the hydrophobic NP more hydrophilic. It is well accepted that hydrophobicity of the NP plays a crucial role in determining its interactions with biological entities. ${ }^{1,22}$ Hydrophobicity of the NPs coated with the PS corona, however, is largely determined by the molecular composition of the corona. As the lipid composition of the corona formed on different NPs is largely identical to the lipid composition of the bulk phase PS (Figure 5c), it is not unexpected that hydrophobicity of the NPs coated with the PS corona tends to be equalized (Table 1). 
Despite the similarity in hydrophobicity, our current (Figure 5d) and previous simulations consistently showed that hydrophobic NPs tend to adsorb more hydrophobic surfactant proteins (i.e., SP-B and/or SP-C). ${ }^{16,26}$ Consequently, the hydrophobic NPs are expected to have a higher inhibitory potential to the biophysical function of natural PS than the hydrophilic NPs. Along this line, our in vitro experiments showed that NPs with increasing hydrophobicity cause a higher degree of surfactant inhibition, likely due to depletion of hydrophobic proteins from the natural PS. ${ }^{15,22,25}$ This finding also supports the strong correlation between the hydrophobicity of NPs and their pulmonary toxicity in vivo. ${ }^{50}$

Third, the PS corona coated on the pristine NP obviously increases the phosphate group density on the NP surface. Because the phosphate group plays a crucial role in the biorecognition of NPs, ${ }^{4}$ the PS corona may lead to enhanced cellular uptake of the NPs. ${ }^{28,30,49}$ Due to differential molecular conformations of the PS corona formed on the hydrophilic and hydrophobic NPs (Figures 2-5), the phosphate group on the Ag-NP shows a larger density than that on the PST-NP (Table $1)$, thus indicating an easier cellular uptake of the hydrophilic NPs than the hydrophobic NPs. This prediction is in line with experimental results by Thorley et al. ${ }^{28}$ These researchers found that hydrophilic modifications and binding with natural PS increase the cellular uptake of PST-NP. Our simulations therefore imply that the different behaviors of cellular update of NPs after acquiring the PS corona is not due to the intrinsic hydrophobicity of the pristine NP (as the PS corona tends to equalize the hydrophobicity of the NP) but likely due to the different phosphate group densities in the PS corona.

It should be noted that although our CGMD simulations provide an insight into the molecular conformation of the PS corona and its impact on nanotoxicology and nano-biotechnology, it is worth putting a caveat on these simulation results. First of all, limited by the computing power, both length and time scales of our simulations are much smaller than those in real experiments. Therefore, any direct quantitative comparison between our simulation results and experimental measurements should be only conducted with caution. Second, ultrastructure and chemical composition of natural PS are very complicated and likely involve within- and between-person variations. Hence, our simulation results can be only used as a general guideline for understanding the formation and structure of the PS corona at the molecular scale. Third, NPs in reality, even before inhalation, are not likely to be pristine. This is especially true for metal particles of high surface free energy. For example, Ag-NP in reality is most likely either partially oxidized or bound with hydroxyl or sulfide ligands adsorbed from the environment. Our CGMD simulations only considered the physicochemical properties of the NP surface instead of the specific chemical composition of the surface.

\section{CONCLUSIONS}

Using CGMD simulations, we studied the interactions between NPs and the natural PS in the alveolar fluid. It was found that regardless of their physicochemical properties, upon contact with the PS, both Ag-NPs and PST-NPs instantly acquire a biomolecular corona that consists of both lipids and proteins. Structure and molecular conformation of the PS corona, however, depend on the hydrophobicity of the pristine NPs, with the hydrophilic Ag-NP forming a bilayered corona and the hydrophobic PST-NP forming a monolayered corona. Quantitative analysis revealed that the lipid composition of the corona formed on different NPs is relatively conserved and is similar to that of the bulk phase PS. Relative abundance of SP-A in the corona of different NPs, however, is significantly higher than that in the bulk phase PS. In contrast to the hydrophilic protein SP-A, the relative abundance of hydrophobic proteins (SP-B and SP-C) is notably affected by the hydrophobicity of the NP. Formation of the PS corona provides the NPs with a physicochemical barrier, equalizes the hydrophobicity of the pristine NPs, and may enhance biorecognition of the NPs. These modifications in physicochemical properties may play a crucial role in affecting the biological identity of the NPs and hence alter their subsequent interactions with cells and other biological entities. Our results suggest that all studies of inhalation nanotoxicology or NPbased pulmonary drug delivery should consider the influence of the PS corona.

\section{METHODS}

Establishment of the Coarse-Grained Models. Coarse-grained molecular dynamics (CGMD) simulations were utilized to capture sufficient details in the molecular scale while allowing the simulation to run in adequate temporal and spatial scales. The widely used MARTINI CG force field was adopted. ${ }^{51}$ Similar to our previous study, the lipid components of the pulmonary surfactant consist of dipalmitoylphosphatidylcholine (DPPC), palmitoyloleoylphosphatidylglycerol (POPG), and cholesterol at a molecular ratio of 14:6:5. ${ }^{26}$ The two hydrophobic surfactant proteins, SP-B and SP-C, were simulated by their corresponding peptides derived from the allatom models obtained from the protein data bank $(\mathrm{PDB})^{52}$ by recognizing their secondary structures using the DSSP program. ${ }^{53}$ The all-atom models were then transferred to the corresponding CG models with the martinize.py script provided by the Martini force field. Mini-B (PDB ID: 2DWF) is a 34-residue peptide composed of the Nand C-terminal helical regions of the full-length 79-residue SP-B. Mini$\mathrm{B}$ has been shown to retain certain activity of the full-length SP-B. ${ }^{54}$ Two tails of the SP-C peptide (PDB ID: 1SPF) has been palmitoylated, which is crucial for its surface activity. ${ }^{55}$

The complete molecular structure of the hydrophilic surfactant protein SP-A is not yet available. However, it is known that SP-A is associated with the PS membranes and interacted with inhaled particles/pathogens primarily through its carbohydrate recognition domain (CRD) ${ }^{38}$ Hence, the fragment of CRD and the neck domain of SP-A (PDB ID: 1R13) were used in our simulations to represent SP-A. Another hydrophilic surfactant protein SP-D, however, was not included in the present simulation because SP-D is not usually associated with the PS membranes. ${ }^{56}$ Detailed all-atom and CG models of the surfactant protein peptides for SP-A, SP-B, and SP-C used in our simulation can be found in Figure S7 of the SI.

The silver (Ag) NPs were constructed with the bead type P4 (because the surface of Ag-NPs is usually covered by a layer of water molecules ${ }^{57}$ ), with each Ag atom represented by one CG bead. The polystyrene (PST) NPs were constructed with $75 \%$ bead type STY and $25 \%$ bead type SCY, as recommended by Rossi et al. ${ }^{58}$ In this model, the benzene ring in the styrene monomer residue was represented by three STY beads, while the alkane chain was represented by one SCY bead. The surface charge density of the NPs was set as $0.06 \mathrm{e} / \mathrm{nm}^{2}$, based on the potentiometric and conductometric titrations by Stone-Masui and Watillon, ${ }^{36}$ and evenly distributed on the surface beads of the NPs.

Process of the CGMD Simulations. All CGMD simulations of natural PS were conducted in a simulation box of $30 \times 30 \times 30 \mathrm{~nm}^{3}$ that contains 1599 CG DPPC, 687 CG POPG, and 573 CG cholesterol molecules, doped with 10 CG mini-B, 10 CG SP-C peptides, and 4 CG SP-A fragments. The NPs and PS molecules were randomly set in a simulation box using the PACKMOL package ${ }^{59}$ and then solvated in a $0.154 \mathrm{M} \mathrm{NaCl}$ aqueous solution to allow selfassembly in the aqueous phase. The initial condition for simulating the self-assembly of PS lipids and proteins is shown in Figure S8 in the SI. 
Periodic boundary conditions were applied in all three dimensions. The system energy was first minimized using the steepest descent algorithm and then the conjugate gradient algorithm, followed by a short-time simulation under NVT ensemble conditions. Simulations were carried in NPT ensembles, using the isotropic Berendsen barostat with a coupling constant of $\tau_{\mathrm{P}}=4$ ps and a system compressibility of 5 $\times 10^{-5} \mathrm{bar}^{-1}$. The time step of simulations was $20 \mathrm{fs}$, and the neighbor list was updated every 10 steps. The temperature was maintained at $310 \mathrm{~K}$ by v-rescale temperature coupling. Simulations in NPT ensembles last at least $750 \mathrm{~ns}$ to ensure the completion of molecular diffusion and interaction. The equilibrium was reached when no new PS molecules were adsorbed to the surface of the NP within a few hundreds of nanoseconds; that is, the number density curves shown in Figure 4 did not change significantly. All simulations were performed using the GROMACS 4.5.4. ${ }^{60}$

Data Analysis and Quantification. The number density of different PS components shown in Figure 4 was evaluated by the Bspline function of $D=N /\left(4 \pi(r+d)^{2} t\right)$ in a series of $0.2 \mathrm{~nm}$ thick spherical shells, where $D$ is the number density, $N$ is the bead number in the shell, $r$ is the radius of the NP, $d$ is the distance from the NP surface, and $t$ is the thickness of the shell. The relative hydrophobicity (Table 1) of the NP (with/without the PS corona) was characterized by the proportion of hydrophobic beads on the NP surface, with zero representing fully hydrophilic and unity representing fully hydrophobic. The surface density of the phosphate group (Table 1 ) on the $\mathrm{NP}$ was evaluated by $N /\left(\pi D^{2}\right)$, where $N$ is the number of PO4-named beads on the outer surface of the corona, and $\pi D^{2}$ is the surface area of the corona.

\section{ASSOCIATED CONTENT}

\section{S Supporting Information}

The Supporting Information is available free of charge on the ACS Publications website at DOI: 10.1021/acsnano.7b01873.

Supplemental simulation results (PDF)

Self-assembly of PS lipids in an aqueous solution (AVI)

Self-assembly of PS lipids and proteins in an aqueous solution (AVI)

Formation of the PS corona on a Ag-NP (AVI)

Formation of the PS corona on a PST-NP (AVI)

\section{AUTHOR INFORMATION}

\section{Corresponding Authors}

*E-mail: guoqing.hu@imech.ac.cn.

*E-mail: yzuo@hawaii.edu.

\section{ORCID}

Yi Y. Zuo: 0000-0002-3992-3238

\section{Notes}

The authors declare no competing financial interest.

\section{ACKNOWLEDGMENTS}

This work was supported by NSFC $(91543125,11572334)$, the CAS Key Research Program of Frontier Sciences (QYZDBSSW-JSC036), and the CAS Strategic Priority Research Program (XDB22040403) to G.H., and NSF Grant Nos. CBET-1604119 and CBET-1236596 to Y.Y.Z. The MD simulations were performed on TianHe-1(A) at the National Supercomputing Center in Tianjin.

\section{REFERENCES}

(1) Nel, A. E.; Madler, L.; Velegol, D.; Xia, T.; Hoek, E. M.; Somasundaran, P.; Klaessig, F.; Castranova, V.; Thompson, M. Understanding Biophysicochemical Interactions at the Nano-Bio Interface. Nat. Mater. 2009, 8, 543-557.

(2) Cedervall, T.; Lynch, I.; Lindman, S.; Berggard, T.; Thulin, E.; Nilsson, H.; Dawson, K. A.; Linse, S. Understanding the Nanoparticle-
Protein Corona Using Methods to Quantify Exchange Rates and Affinities of Proteins for Nanoparticles. Proc. Natl. Acad. Sci. U. S. A. 2007, 104, 2050-2055.

(3) Lynch, I.; Dawson, K. A. Protein-Nanoparticle Interactions. Nano Today 2008, 3, 40-47.

(4) Monopoli, M. P.; Aberg, C.; Salvati, A.; Dawson, K. A. Biomolecular Coronas Provide the Biological Identity of Nanosized Materials. Nat. Nanotechnol. 2012, 7, 779-786.

(5) Aggarwal, P.; Hall, J. B.; McLeland, C. B.; Dobrovolskaia, M. A.; McNeil, S. E. Nanoparticle Interaction with Plasma Proteins as It Relates to Particle Biodistribution, Biocompatibility and Therapeutic Efficacy. Adv. Drug Delivery Rev. 2009, 61, 428-437.

(6) Lesniak, A.; Fenaroli, F.; Monopoli, M. R.; Aberg, C.; Dawson, K. A.; Salvati, A. Effects of the Presence or Absence of a Protein Corona on Silica Nanoparticle Uptake and Impact on Cells. ACS Nano 2012, 6, 5845-5857.

(7) Hu, W.; Peng, C.; Lv, M.; Li, X.; Zhang, Y.; Chen, N.; Fan, C.; Huang, Q. Protein Corona-Mediated Mitigation of Cytotoxicity of Graphene Oxide. ACS Nano 2011, 5, 3693-3700.

(8) Lunov, O.; Syrovets, T.; Loos, C.; Beil, J.; Delacher, M.; Tron, K.; Nienhaus, G. U.; Musyanovych, A.; Mailander, V.; Landfester, K.; Simmet, T. Differential Uptake of Functionalized Polystyrene Nanoparticles by Human Macrophages and a Monocytic Cell Line. ACS Nano 2011, 5, 1657-1669.

(9) Qiao, R.; Roberts, A. P.; Mount, A. S.; Klaine, S. J.; Ke, P. C. Translocation of C60 and Its Derivatives Across a Lipid Bilayer. Nano Lett. 2007, 7, 614-619.

(10) Setyawati, M. I.; Tay, C. Y.; Docter, D.; Stauber, R. H.; Leong, D. T. Understanding and Exploiting Nanoparticles' Intimacy with the Blood Vessel and Blood. Chem. Soc. Rev. 2015, 44, 8174-8199.

(11) Milani, S.; Baldelli Bombelli, F.; Pitek, A. S.; Dawson, K. A.; Radler, J. Reversible Versus Irreversible Binding of Transferrin to Polystyrene Nanoparticles: Soft and Hard Corona. ACS Nano 2012, 6, 2532-2541.

(12) Lundqvist, M.; Stigler, J.; Elia, G.; Lynch, I.; Cedervall, T.; Dawson, K. A. Nanoparticle Size and Surface Properties Determine the Protein Corona with Possible Implications for Biological Impacts. Proc. Natl. Acad. Sci. U. S. A. 2008, 105, 14265-14270.

(13) Tenzer, S.; Docter, D.; Rosfa, S.; Wlodarski, A.; Kuharev, J.; Rekik, A.; Knauer, S. K.; Bantz, C.; Nawroth, T.; Bier, C.; Sirirattanapan, J.; Mann, W.; Treuel, L.; Zellner, R.; Maskos, M.; Schild, H.; Stauber, R. H. Nanoparticle Size is a Critical Physicochemical Determinant of the Human Blood Plasma Corona: a Comprehensive Quantitative Proteomic Analysis. ACS Nano 2011, 5, $7155-7167$.

(14) Muhlfeld, C.; Rothen-Rutishauser, B.; Blank, F.; Vanhecke, D.; Ochs, M.; Gehr, P. Interactions of Nanoparticles with Pulmonary Structures and Cellular Responses. Am. J. Physiol. Lung Cell Mol. Physiol. 2008, 294, L817-L829.

(15) Fan, Q.; Wang, Y. E.; Zhao, X.; Loo, J. S.; Zuo, Y. Y. Adverse Biophysical Effects of Hydroxyapatite Nanoparticles on Natural Pulmonary Surfactant. ACS Nano 2011, 5, 6410-6416.

(16) Hu, G.; Jiao, B.; Shi, X.; Valle, R. P.; Fan, Q.; Zuo, Y. Y. Physicochemical Properties of Nanoparticles Regulate Translocation Across Pulmonary Surfactant Monolayer and Formation of Lipoprotein Corona. ACS Nano 2013, 7, 10525-10533.

(17) Raesch, S. S.; Tenzer, S.; Storck, W.; Rurainski, A.; Selzer, D.; Ruge, C. A.; Perez-Gil, J.; Schaefer, U. F.; Lehr, C. M. Proteomic and Lipidomic Analysis of Nanoparticle Corona upon Contact with Lung Surfactant Reveals Differences in Protein, but Not Lipid Composition. ACS Nano 2015, 9, 11872-11885.

(18) Zuo, Y. Y.; Veldhuizen, R. A.; Neumann, A. W.; Petersen, N. O.; Possmayer, F. Current Perspectives in Pulmonary SurfactantInhibition, Enhancement and Evaluation. Biochim. Biophys. Acta, Biomembr. 2008, 1778, 1947-1977.

(19) Whitsett, J. A.; Weaver, T. E. Hydrophobic Surfactant Proteins in Lung Function and Disease. N. Engl. J. Med. 2002, 347, 2141-2148.

(20) Hickling, T. P.; Clark, H.; Malhotra, R.; Sim, R. B. Collectins and Their Role in Lung Immunity. J. Leukocyte Biol. 2003, 75, 27-33. 
(21) Haagsman, H. P. Structural and Functional Aspects of the Collectin SP-A. Immunobiology 2002, 205, 476-489.

(22) Valle, R. P.; Huang, C. L.; Loo, J. S. C.; Zuo, Y. Y. Increasing Hydrophobicity of Nanoparticles Intensifies Lung Surfactant Film Inhibition and Particle Retention. ACS Sustainable Chem. Eng. 2014, 2, $1574-1580$

(23) Beck-Broichsitter, M.; Ruppert, C.; Schmehl, T.; Günther, A.; Seeger, W. Biophysical Inhibition of Synthetic vs. Naturally-Derived Pulmonary Surfactant Preparations by Polymeric Nanoparticles. Biochim. Biophys. Acta, Biomembr. 2014, 1838, 474-481.

(24) Sachan, A. K.; Galla, H. J. Understanding the Mutual Impact of Interaction Between Hydrophobic Nanoparticles and Pulmonary Surfactant Monolayer. Small 2014, 10, 1069-1075.

(25) Valle, R. P.; Wu, T.; Zuo, Y. Y. Biophysical Influence of Airborne Carbon Nanomaterials on Natural Pulmonary Surfactant. ACS Nano 2015, 9, 5413-5421.

(26) Hu, Q.; Jiao, B.; Shi, X.; Valle, R. P.; Zuo, Y. Y.; Hu, G. Effects of Graphene Oxide Nanosheets on the Ultrastructure and Biophysical Properties of the Pulmonary Surfactant Film. Nanoscale 2015, 7, 18025-18029.

(27) Kapralov, A. A.; Feng, W. H.; Amoscato, A. A.; Yanamala, N.; Balasubramanian, K.; Winnica, D. E.; Kisin, E. R.; Kotchey, G. P.; Gou, P.; Sparvero, L. J.; Ray, P.; Mallampalli, R. K.; Klein-Seetharaman, J.; Fadeel, B.; Star, A.; Shvedova, A. A.; Kagan, V. E. Adsorption of Surfactant Lipids by Single-Walled Carbon Nanotubes in Mouse Lung upon Pharyngeal Aspiration. ACS Nano 2012, 6, 4147-4156.

(28) Thorley, A. J.; Ruenraroengsak, P.; Potter, T. E.; Tetley, T. D. Critical Determinants of Uptake and Translocation of Nanoparticles by the Human Pulmonary Alveolar Epithelium. ACS Nano 2014, 8, $11778-11789$

(29) Vranic, S.; Garcia-Verdugo, I.; Darnis, C.; Sallenave, J. M.; Boggetto, N.; Marano, F.; Boland, S.; Baeza-Squiban, A. Internalization of $\mathrm{SiO}(2)$ Nanoparticles by Alveolar Macrophages and Lung Epithelial Cells and Its Modulation by the Lung Surfactant Substitute Curosurf. Environ. Sci. Pollut. Res. 2013, 20, 2761-2770.

(30) Sund, J.; Alenius, H.; Vippola, M.; Savolainen, K.; Puustinen, A. Proteomic Characterization of Engineered Nanomaterial-Protein Interactions in Relation to Surface Reactivity. ACS Nano 2011, 5, 4300-4309.

(31) Whitwell, H.; Mackay, R.-M.; Elgy, C.; Morgan, C.; Griffiths, M.; Clark, H.; Skipp, P.; Madsen, J. Nanoparticles in the Lung and Their Protein Corona: the Few Proteins that Count. Nanotoxicology 2016, 10, 1385-1394.

(32) Lopez-Rodriguez, E.; Perez-Gil, J. Structure-Function Relationships in Pulmonary Surfactant Membranes: from Biophysics to Therapy. Biochim. Biophys. Acta, Biomembr. 2014, 1838, 1568-1585.

(33) Zhang, H.; Wang, Y. E.; Neal, C. R.; Zuo, Y. Y. Differential Effects of Cholesterol and Budesonide on Biophysical Properties of Clinical Surfactant. Pediatr. Res. 2012, 71, 316-323.

(34) Bernhard, W.; Mottaghian, J.; Gebert, A.; Rau, G. A.; von der Hardt, H.; Poets, C. F. Commercial Versus Native Surfactants. Surface Activity, Molecular Components, and the Effect of Calcium. Am. J. Respir. Crit. Care Med. 2000, 162, 1524-1533.

(35) Wang, Z.; Xia, T.; Liu, S. Mechanisms of Nanosilver-Induced Toxicological Effects: More Attention Should Be Paid to Its Sublethal Effects. Nanoscale 2015, 7, 7470-7481.

(36) Stone-Masui, J.; Watillon, A. Characterization of Surface Charge on Polystyrene Latices. J. Colloid Interface Sci. 1975, 52, 479-503.

(37) Cruz, A.; Casals, C.; Plasencia, I.; Marsh, D.; Perez-Gil, J. Depth Profiles of Pulmonary Surfactant Protein B in Phosphatidylcholine Bilayers, Studied by Fluorescence and Electron Spin Resonance Spectroscopy. Biochemistry 1998, 37, 9488-9496.

(38) Palaniyar, N.; Ikegami, M.; Korfhagen, T.; Whitsett, J.; McCormack, F. X. Domains of Surfactant Protein A that Affect Protein Oligomerization, Lipid Structure and Surface Tension. Comp. Biochem. Physiol., Part A: Mol. Integr. Physiol. 2001, 129, 109-127.

(39) Ruge, C. A.; Schaefer, U. F.; Herrmann, J.; Kirch, J.; Canadas, O.; Echaide, M.; Perez-Gil, J.; Casals, C.; Muller, R.; Lehr, C. M. The
Interplay of Lung Surfactant Proteins and Lipids Assimilates the Macrophage Clearance of Nanoparticles. PLoS One 2012, 7, e40775.

(40) McKenzie, Z.; Kendall, M.; Mackay, R. M.; Whitwell, H.; Elgy, C.; Ding, P.; Mahajan, S.; Morgan, C.; Griffiths, M.; Clark, H.; Madsen, J. Surfactant Protein A (SP-A) Inhibits Agglomeration and Macrophage Uptake of Toxic Amine Modified Nanoparticles. Nanotoxicology 2015, 9, 952-962.

(41) Ruge, C. A.; Kirch, J.; Canadas, O.; Schneider, M.; Perez-Gil, J.; Schaefer, U. F.; Casals, C.; Lehr, C. M. Uptake of Nanoparticles by Alveolar Macrophages is Triggered by Surfactant Protein A. Nanomedicine 2011, 7, 690-693.

(42) Salvador-Morales, C.; Townsend, P.; Flahaut, E.; Venien-Bryan, C.; Vlandas, A.; Green, M. L. H.; Sim, R. B. Binding of Pulmonary Surfactant Proteins to Carbon Nanotubes; Potential for Damage to Lung Immune Defense Mechanisms. Carbon 2007, 45, 607-617.

(43) Schulze, C.; Schaefer, U. F.; Ruge, C. A.; Wohlleben, W.; Lehr, C. M. Interaction of Metal Oxide Nanoparticles with Lung Surfactant Protein A. Eur. J. Pharm. Biopharm. 2011, 77, 376-383.

(44) Seiffert, J.; Hussain, F.; Wiegman, C.; Li, F.; Bey, L.; Baker, W.; Porter, A.; Ryan, M. P.; Chang, Y.; Gow, A.; Zhang, J.; Zhu, J.; Tetley, T. D.; Chung, K. F. Pulmonary Toxicity of Instilled Silver Nanoparticles: Influence of Size, Coating and Rat Strain. PLoS One 2015, 10, e0119726.

(45) Takenaka, S.; Karg, E.; Roth, C.; Schulz, H.; Ziesenis, A.; Heinzmann, U.; Schramel, P.; Heyder, J. Pulmonary and Systemic Distribution of Inhaled Ultrafine Silver Particles in Rats. Environ. Health Persp. 2001, 109, 547-551.

(46) Wang, X.; Ji, Z.; Chang, C. H.; Zhang, H.; Wang, M.; Liao, Y.-P.; Lin, S.; Meng, H.; Li, R.; Sun, B.; Winkle, L. V.; Pinkerton, K. E.; Zink, J. I.; Xia, T.; Nel, A. E. Use of Coated Silver Nanoparticles to Understand the Relationship of Particle Dissolution and Bioavailability to Cell and Lung Toxicological Potential. Small 2014, 10, 385-398.

(47) Chen, Y.; Wang, Z.; Xu, M.; Wang, X.; Liu, R.; Liu, Q.; Zhang, Z.; Xia, T.; Zhao, J.; Jiang, G.; Xu, Y.; Liu, S. Nanosilver Incurs an Adaptive Shunt of Energy Metabolism Mode to Glycolysis in Tumor and Nontumor Cells. ACS Nano 2014, 8, 5813-5825.

(48) Leo, B. F.; Chen, S.; Kyo, Y.; Herpoldt, K. L.; Terrill, N. J.; Dunlop, I. E.; McPhail, D. S.; Shaffer, M. S.; Schwander, S.; Gow, A.; Zhang, J.; Chung, K. F.; Tetley, T. D.; Porter, A. E.; Ryan, M. P. The Stability of Silver Nanoparticles in a Model of Pulmonary Surfactant. Environ. Sci. Technol. 2013, 47, 11232-11240.

(49) Theodorou, I. G.; Ruenraroengsak, P.; Gow, A.; Schwander, S.; Zhang, J. J.; Chung, K. F.; Tetley, T. D.; Ryan, M. P.; Porter, A. E. Effect of Pulmonary Surfactant on the Dissolution, Stability and Uptake of Zinc Oxide Nanowires by Human Respiratory Epithelial Cells. Nanotoxicology 2016, 10, 1351-1362.

(50) Dailey, L. A.; Hernandez-Prieto, R.; Casas-Ferreira, A. M.; Jones, M. C.; Riffo-Vasquez, Y.; Rodriguez-Gonzalo, E.; Spina, D.; Jones, S. A.; Smith, N. W.; Forbes, B.; Page, C.; Legido-Quigley, C. Adenosine Monophosphate is Elevated in the Bronchoalveolar Lavage Fluid of Mice with Acute Respiratory Toxicity Induced by Nanoparticles with High Surface Hydrophobicity. Nanotoxicology 2015, 9, 106-115.

(51) Marrink, S. J.; Risselada, H. J.; Yefimov, S.; Tieleman, D. P.; de Vries, A. H. The MARTINI Force Field: Coarse Grained Model for Biomolecular Simulations. J. Phys. Chem. B 2007, 111, 7812-7824.

(52) Bernstein, F. C.; Koetzle, T. F.; Williams, G. J. B.; Meyer, E. F.; Brice, M. D.; Rodgers, J. R.; Kennard, O.; Shimanouchi, T.; Tasumi, M. The Protein Data Bank. A Computer-Based Archival File for Macromolecular Structures. Eur. J. Biochem. 1977, 80, 319-324.

(53) Touw, W. G.; Baakman, C.; Black, J.; te Beek, T. A.; Krieger, E.; Joosten, R. P.; Vriend, G. A Series of PDB-Related Databanks for Everyday Needs. Nucleic Acids Res. 2015, 43, D364-D368.

(54) Sarker, M.; Waring, A. J.; Walther, F. J.; Keough, K. M.; Booth, V. Structure of Mini-B, a Functional Fragment of Surfactant Protein B, in Detergent Micelles. Biochemistry 2007, 46, 11047-11056.

(55) Baumgart, F.; Ospina, O. L.; Mingarro, I.; Rodriguez-Crespo, I.; Perez-Gil, J. Palmitoylation of Pulmonary Surfactant Protein SP-C is Critical for Its Functional Cooperation with SP-B to Sustain 
Compression/Expansion Dynamics in Cholesterol-Containing Surfactant Films. Biophys. J. 2010, 99, 3234-3243.

(56) Crouch, E. C. Structure, Biologic Properties, and Expression of Surfactant Protein D (SP-D). Biochim. Biophys. Acta, Mol. Basis Dis. 1998, 1408, 278-289.

(57) Hughes, Z. E.; Wright, L. B.; Walsh, T. R. Biomolecular Adsorption at Aqueous Silver Interfaces: First-Principles Calculations, Polarizable Force-Field Simulations, and Comparisons with Gold. Langmuir 2013, 29, 13217-13229.

(58) Rossi, G.; Monticelli, L.; Puisto, S. R.; Vattulainen, I.; AlaNissila, T. Coarse-Graining Polymers with the MARTINI Force-Field: Polystyrene as a Benchmark Case. Soft Matter 2011, 7, 698-708.

(59) Martinez, L.; Andrade, R.; Birgin, E. G.; Martinez, J. M. PACKMOL: a Package for Building Initial Configurations for Molecular Dynamics Simulations. J. Comput. Chem. 2009, 30, 21572164.

(60) Hess, B.; Kutzner, C.; van der Spoel, D.; Lindahl, E. GROMACS 4: Algorithms for Highly Efficient, Load-Balanced, and Scalable Molecular Simulation. J. Chem. Theory Comput. 2008, 4, 435-447. 